\title{
Beneficial reciprocal effects of bone marrow stromal cells and Schwann cells from adult rats in a dynamic co-culture system in vitro without intercellular contact
}

\author{
LI-NA ZHOU, XIAO-JUN CUI, KAI-XIN SU, XIAO-HONG WANG and JIN-HUA GUO
}

Department of Anatomy, Guangdong Medical University, Zhanjiang, Guangdong 524023, P.R. China

Received December 29, 2014; Accepted June 11, 2015

DOI: $10.3892 / \mathrm{mmr} .2015 .4016$

\begin{abstract}
In order to examine how implanted bone marrow stromal cells (BMSCs) encourage peripheral nerve regeneration, the present study investigated the interaction of BMSCs and Schwann cells (SCs) using an indirect in vitro co-culture model. SCs and BMSCs were obtained from adult Sprague-Dawley rats. The passaged BMSCs were CD29- and CD44-positive but CD45-negative and were co-cultured with the primary SCs using a Millicell system, which allows BMSCs and SCs to grow in the same culture medium but without direct contact. Expression of the typical SC markers S-100 and glial fibrillary acidic protein (GFAP) of the treated BMSCs as well as the proliferation capacity of the co-cultured SCs was evaluated by immunocytochemical staining on the $3 \mathrm{rd}$ and 5th day of co-culture. Immunocytochemical staining showed that $>75 \%$ of the BMSCs in the indirect co-culture model were GFAP- and S-100-positive on the 3rd and 5th day after co-culture, as opposed to $<5 \%$ of the BMSCs in the control group. On the 3rd day after co-culture, only a few co-cultured BMSCs showed the typical SC-like morphology, while most BMSCs still kept their native appearance. By contrast, on the 5th day after co-culture, almost all of the co-cultured BMSCs appeared with the typical SC-like morphology. Furthermore, $70.71 \%$ of the SCs in the indirect co-culture model were S-100-positive on the 5th day of co-culture, as opposed to $>30.43 \%$ of the SCs in the control group. These results indicated that BMSCs may interact synergistically with SCs with regard to promoting peripheral nerve regeneration.
\end{abstract}

\section{Introduction}

Schwann cells (SCs) have a critical role in peripheral nerve regeneration $(1,2)$. Experimentally, SCs transplantation has

Correspondence to: Mrs. Li-Na Zhou, Department of Anatomy, Guangdong Medical University, 2 Xiashan Civilization Road, Zhanjiang, Guangdong 524023, P.R. China

E-mail:21456783@qq.com

Key words: bone marrow stromal cells, Schwann cells, co-culture, differentiation, proliferation been shown to enhance axon outgrowth in vitro (3) as well as in vivo $(4,5)$. However, achieving autologous SCs requires sacrifice of a donor nerve, leading to functional deficits at the donor site. The limitations of autologous SC grafting have motivated efforts to seek for alternatives. The ideal seed cell should be easily accessible, proliferate rapidly in vitro, possess multipotential differentiation properties and successfully integrate into host tissue, with non- or hypo-immunogenicity $(6,7)$. Bone marrow stromal cells (BMSCs) can meet the above requirements and are considered as an alternative seed cell. They are a population of non-hematopoietic multipotent somatic stem cells harbored in the bone marrow, which are defined by their ability to adhere to plastic surfaces and by a panel of positive and negative cell surface markers $(8,9)$. Under specific conditions, these cells can differentiate into multiple mesodermal cell lineages, including chondrocytes, osteocytes and adipocytes (8-10) and into neuronal phenotypes, including astrocytes, oligodendrocytes, microglia and neurons $(9,11-14)$. Previous studies have reported that BMSCs derived from multiple mammalian species can be transdifferentiated into cells with SC phenotype in the presence of inducing agents or growth factor cocktails (15-20). More importantly, the implanted BMSCs have been proved to promote axonal regeneration and functional recovery in peripheral nerve repair (20-23). To contribute to the understanding of how BMSCs are involved in peripheral nerve regeneration, the present study hypothesized that BMSCs may exert their efficacy not only via transdifferentiation into SCs-like cells, but through indirect influence of the cellular behavior of SCs. To test this hypothesis, the present study focused on examining the cellular interactions of BMSCs and SCs in vitro by indirect co-culture in vitro.

\section{Materials and methods}

Animals. Adult Sprague-Dawley rats (200-250 g) were obtained from the Laboratory Animal Centre of Guangdong Medical University (Dongguan, China). All rats were maintained under standard housing conditions $\left(25 \pm 2^{\circ} \mathrm{C}\right.$, humidity of $50 \pm 5 \%$ and a $12 \mathrm{~h}$ light-dark cycle) with ad libitum access to food and water. All animals were anaesthetized with $10 \%$ chloral hydrate $[0.1 \mathrm{~g} / \mathrm{ml}$ intraperitoneally (i.p.)]. All surgical procedures conformed to the Chinese National Health and Medical Research Council (NHMRC) animal ethics guidelines and 
were approved by the Guangdong Medical University Animal Experimentation Ethics Committee. All animals were given $60 \mathrm{mg}$ benzylpenicillin (BioBasic, Shanghai, China) subcutaneously (s.c.) and allowed to recover from the anaesthesia prior to returning to their cages.

SCs cultures. Schwann cells (SCs) were enriched from pre-degenerated sciatic nerves of adult Sprague-Dawley rats as described in a previous study by our group (24). The protocol is based on SC proliferation after Wallerian degeneration, combined use of collagenase/dispase and mitogen in culture medium. In brief, the sciatic nerves were ligated to allow the nerves to pre-degenerate. The pre-degenerated nerves $\sim 20 \mathrm{~mm}$ in length were cut into $1-\mathrm{mm}$ pieces. The minced nerves were enzymatically dissociated using an enzyme mixture of $0.05 \%$ collagenase/dispase (Roche, Basel, Switzerland) in Dulbecco's modified Eagle's medium (DMEM)/F12 (Gibco-BRL, Invitrogen Life Technologies, Carlsbad, CA, USA) for $5.5 \mathrm{~h}$ at $37^{\circ} \mathrm{C}$. Following dissociation, the cell suspension was centrifuged at $200 \mathrm{x} g$ for $10 \mathrm{~min}$. The dissociated SCs were re-suspended and cultured in DMEM/F12 supplemented with $10 \%$ fetal bovine serum (FBS; Kibbutz BeitHaemek, Israel), 1\% penicillin/streptomycin (BioBasic), $2 \mu \mathrm{M}$ forskolin $(0.8 \mu \mathrm{g} / \mathrm{l}$; Sigma-Aldrich, St Louis, $\mathrm{MO}, \mathrm{USA}$ ) and $20 \mu \mathrm{g} / \mathrm{l}$ bovine pituitary extract (Sigma-Aldrich). After the cultures reached confluence, they were used for graft procedures.

BMSC culture. BMSCs were obtained from adult rat femurs. Isolation of BMSCs was accomplished using a modification of a previously published method (24). The fresh complete bone marrow was flushed with $5 \mathrm{ml}$ DMEM/F12 containing 10\% FBS, $2 \mu \mathrm{M}$ L-glutamine (Gibco-BRL), $10 \mu \mathrm{g} / \mathrm{ml}$ epidermal growth factor (EGF; Peprotech, Rocky Hill, NJ, USA) and $1 \%$ penicillin/streptomycin. The homogeneous cell suspensions were plated in $25-\mathrm{cm}^{2}$ flasks, and the cells were incubated at $37^{\circ} \mathrm{C}$ in $5 \% \mathrm{CO}_{2}$ for $48 \mathrm{~h}$, The non-adherent cells were then removed by washing with phosphate-buffered saline (PBS) and the media were replaced three times per week. When the cells reached $80 \%$ confluence, they were passaged, split and re-plated. Passage (P)-10 cells were prepared for graft procedures.

Flow-cytometric analysis of cultured BMSCs. The BMSCs at P13 were analyzed by fluorescence-activated cell sorting (FACS) as described previously (24). Briefly, BMSCs at P12 were detached in $0.25 \%$ trypsin and $0.02 \%$ EDTA. The $50-\mathrm{ml}$ suspensions at a concentration of $10^{6} / \mathrm{ml}$ were used as the test set and incubated with anti-CD29-fluorescein isothiocyanate (FITC) (1:100 dilution; LabVision, Thermo Fisher Scientific, Waltham, MA, USA), CD44-FITC (1:100; Santa Cruz Biotechnology, Inc, Dallas, TX, USA) or CD45-PE-Cy5 (1:50; LabVision), under the exclusion of light at room temperature for $20 \mathrm{~min}$. Flow-cytometric analyses were performed on a FACS flow cytometer (BD FACSCalibur; Becton Dickinson, Franklin Lakes, NJ, USA) equipped with an argon-ion laser tuned at $488 \mathrm{~nm}$. Data acquisition and analysis were performed using Lysis II software (Becton Dickinson).

Co-culture of adult BMSCs and SCs. The Millcell-HA system, which consisted of a lower six-well plate and an upper culture plate insert with an HA membrane (Millipore, Billerica, MA, USA), was used for co-culture of the BMSCs and SCs in the present study as previously described $(24,25)$. This HA membrane prevented intercellular contact between the SCs and BMSCs (25), allowing the upper and the lower cells to exist in a dynamically synchronous environment, as the pore size $(0.45 \mathrm{~mm})$ of the membrane was small relative to the cell body size of either BMSCs or SCs. In the present study, two co-culture models were established: One model was used to assess the proliferation of SCs, with the SCs seeded into the lower six-well plate at a density of $2 \times 10^{5}$ cells $/ 9.6 \mathrm{~cm}^{2}$ and BMSCs were grown on the upper HA membrane at a density of $1 \times 10^{5}$ cells $/ 9.6 \mathrm{~cm}^{2}$; the other model was used to assess the differentiation of BMSCs by seeding BMSCs into the lower six-well plate at a density of $1 \times 10^{5}$ cells $/ 9.6 \mathrm{~cm}^{2}$, with SCs grown on the upper HA membrane at a density of $2 \times 10^{5}$ cells $/ 9.6 \mathrm{~cm}^{2}$. The co-culture medium was DMEM/F12 containing 10\% FBS, $2 \mathrm{mM}$ L-glutamine, $10 \mu \mathrm{g} / \mathrm{ml} \mathrm{EGF}$ and $1 \%$ penicillin/streptomycin. For each co-culture model, control cultures of BMSCs or SCs were prepared using identical systems which did not contain any cells on the upper membrane. The duration of the co-culture and control cultures was 2, 3 and 5 days.

Fixation and staining of the Millicell membrane. The Millicell-HA insert was removed from the six-well plate at the 2nd and 5th days of co-culture and gently washed with PBS to remove the growth media. The HA membrane with attached cells was removed using a cork borer and fixed for $15 \mathrm{~min}$ with $3 \%$ glutaraldehyde in PBS. After gentle rinsing with deionized water twice, the membrane was incubated for $15 \mathrm{~min}$ using hematoxylin solution (Beijing CellChip Biotechnology Co., Ltd., Beijing, China) at room temperature. The HA membrane was then rinsed with deionized water to remove the stain and then de-stained by adding $0.5 \%$ hydrochloric acid in $70 \%$ ethanol for 2-3 min. After gentle rinsing with deionized water twice, the HA membrane was incubated until a uniform blue color was observed on the membrane by adding diluted ammonium hydroxide. The membrane was then mounted on a slide in neutral gum. The morphology of the cultured cells was observed under a microscope (DFC350FX/DMIRB; Leica Microsystems, Wetzlar, Germany).

Immunofluorescence and nuclear staining. An immunofluorescence assay was performed as reported in a previous study by our group (24). The cultured cells in the six-well plates, which had been rinsed and fixed, were incubated overnight at $4^{\circ} \mathrm{C}$ with the primary antibodies [S-100 (cat. no. HPA015768; 1:4,000 dilution; Sigma-Aldrich) and GFAP (cat. no. G3893; 1:2,000; Sigma-Aldrich)]. The samples were then incubated with fluorescein-conjugated secondary antibodies (cat. nos. SAB3700827 and SAB3701170; Sigma-Aldrich) for $2 \mathrm{~h}$ at room temperature. The primary antibodies were omitted in the negative control. The incubated cells were then re-stained with Hoechst 33258 nuclear marker (5 mg/l; Sigma-Aldrich). The labelled cells were observed under a fluorescence microscope (DFC350FX/DMIRB; Leica Microsystems). To verify the proliferation of cultured SCs, SCs showing S-100-positive, GFAP-positive or Hoechst 33258 labelling were counted in three wells, which was repeated four times. Each of the four repeats was from a different culture, with each culture in turn being derived from a different animal. 

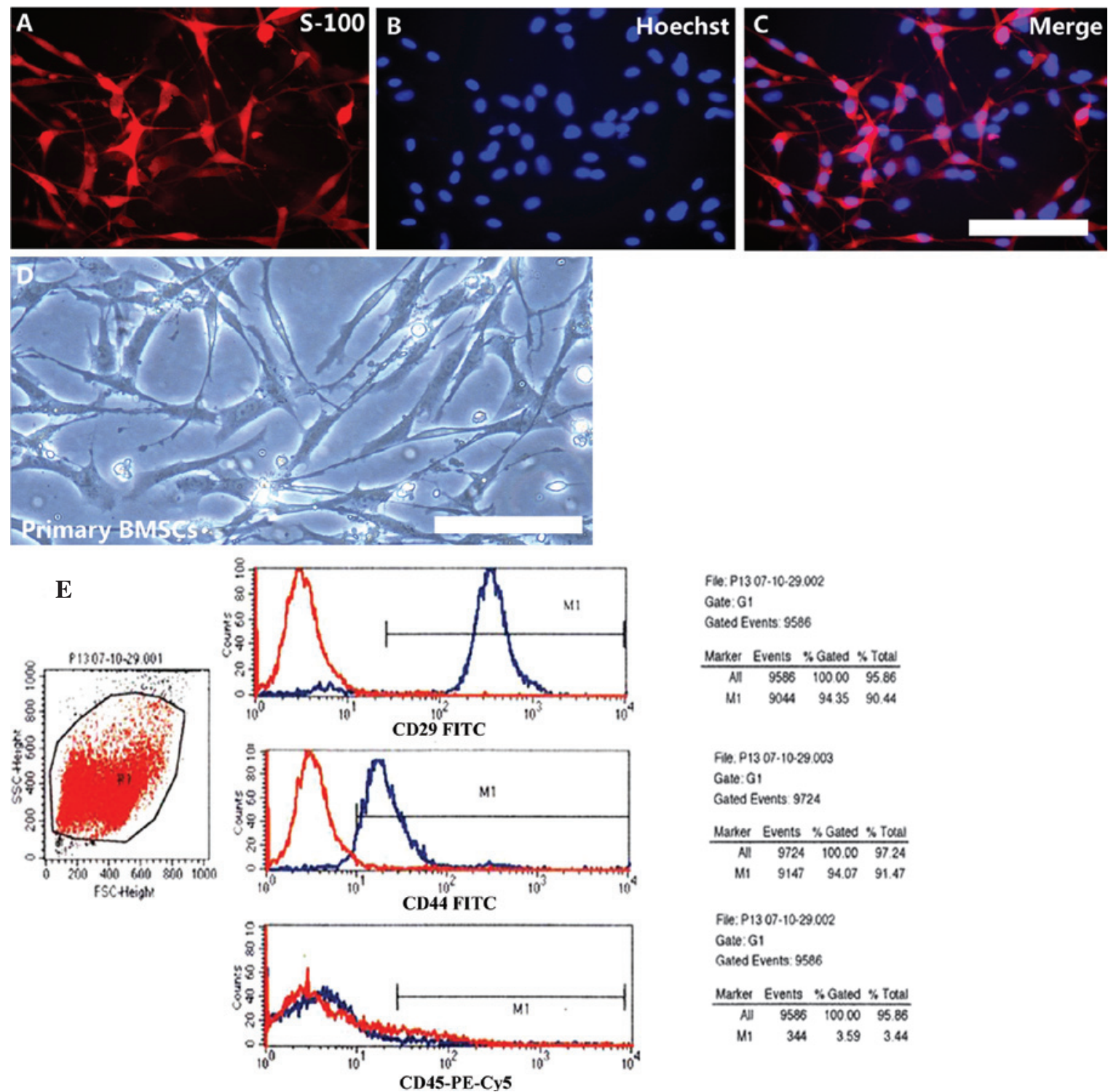

Figure 1. Morphology of adult SCs and BMSCs in vitro. (A-C) Immunofluorescence staining of adult primary SCs: (A) S-100 (red); (B) Hoechst 33258 (blue); (C) merged (scale bar, $50 \mu \mathrm{m}$ ). (D) Phase-contrast images of adult primary BMSCs (scale bar, 100 $\mu \mathrm{m}$ ). (E) Flow-cytometric analysis showed CD29- and CD44-positive but CD45-negative signals in P13 BMSCs of adult rats (blue, assay group; red, control group). BMSC, bone marrow stromal cell; SC, Schwann cell; FITC, fluorescein isothiocyanate; PE, phycoerythrin; FSC, forward scatter; SSC, side scatter.

Statistical analysis. All statistical analyses were performed using SPSS 15.0 (SPSS Inc., Chicago, IL, USA). The data are presented as the mean \pm standard deviation. Comparisons were made between the groups using Mann-Whitney U-test. $\mathrm{P}<0.05$ was considered to indicate a statistically significant difference.

\section{Results}

Characteristics of adult primary SCs and BMSCs in vitro prior to co-culture. In the present study, the primary adult SCs were confluent at 4-5 days of culture. Most of the S-100-positive SCs had typical spindle-shaped bodies with two slender processes. Only a few cells showed triangular soma with three projections (Fig. 1A). The proportion of S-100-positive SCs was $69 \pm 3.8 \%$ at the time of confluence (Fig. 1B and C). At 6-7 days, the adult primary BMSCs were confluent with cells being heterogeneous in morphology
(Fig. 1D). They were easily expanded to up to 15 passages, maintaining the undifferentiated state as fibroblastic morphology. Flow-cytometric analysis showed that the P13 BMSCs were positive for the well-defined BMSC markers CD29 and CD44, but negative for the haematopoietic surface antigen CD45 (Fig. 1E).

SC proliferation is promoted in the BMSC co-culture system. As shown by double labelling with S-100 (Fig. 2A) and Hoechst 33258 (Fig. 2B) on the fifth day of co-culture, SCs co-cultured with BMSCs exhibited a clear spindle-shaped or triangular soma with two or three slender processes (Fig. 2A and C). Either S-100-labelled cells (Fig. 2A and D) or Hoechst 33258-labelled nuclei (Fig. 2B and E) were obviously more numerous in the BMSCs co-culture system than in the acellular control cultures (Fig. 2C and F). The quantitative study showed that the number of S-100-positive SCs as a percentage of the number of 

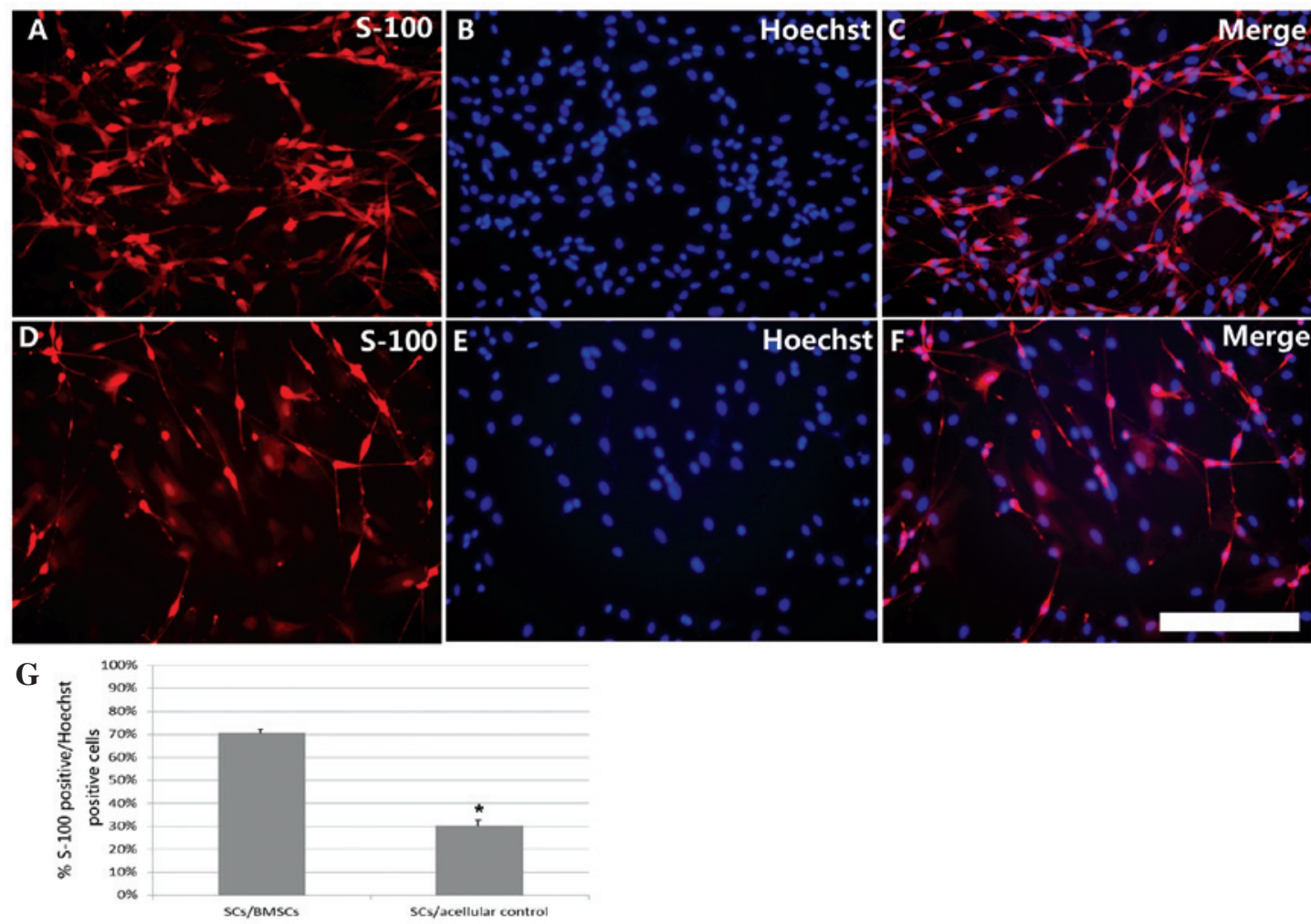

Figure 2. Induction of SC proliferation by diffusible factors of BMSCs. The proliferation of SCs co-cultured with (A-C) BMSCs and (D-F) control was assessed by (A and D) S-100 (red) immunofluorescence and (B and E) Hoechst 33258 nuclear staining (blue) (scale bar, $100 \mu \mathrm{m}$ ). (G) The number of S-100-positive cells as a percentage of the number of Hoechst-positive nuclei was averaged from three wells and repeated four times. Each of the four repeats was from a different culture, with each culture in turn being derived from a different animal. Quantitative analysis showed that the number of SCs co-cultured with BMSCs $(70.71 \pm 1.24 \%, \mathrm{n}=6)$ was significantly higher than that of the control SCs $(30.43 \pm 2.23 \%, \mathrm{n}=6)(\mathrm{P}<0.05)$. BMSC, bone marrow stromal cell; SC, Schwann cell.

Hoechst-labelled nuclei in the BMSC co-culture was significantly higher than that in the acellular control culture, after 5 days $(70.71 \pm 1.24$ vs. $30.43 \pm 2.23 \%$; $\mathrm{P}<0.05)$ (Fig. 2G).

BMSC differentiation is induced in the SC co-culture system. Most of the BMSCs in the co-culture system were GFAP- and S-100-positive on the third and fifth day of co-culture, while the BMSCs in the control culture system hardly showed any GFAP- and S-100-positive populations. On the third day after co-culture, the BMSCs in the co-culture system became dense and grew in a longitudinal, parallel fashion (Fig. 3A and B). Only a few co-cultured BMSCs showed spindle-shaped or triangular soma with two or three slender processes (Fig. 3), the typical morphology of glial differentiation, and most of the co-cultured BMSCs still kept their native appearance. By contrast, on the 5th day after co-culture, almost all of the co-cultured BMSCs appeared with typical SC-like morphology, featuring spindle-shaped or triangular soma with two or three slender processes on the 5th day of co-culture (Fig. 4), the characteristic morphology of glial differentiation. The densities of co-cultured BMSCs were significantly higher in SC co-cultures than in acellular control cultures, whether on the 3 rd or 5th day of co-culture, as shown by S-100 (Fig. 3A and E, and Fig. 4A and E), GFAP (Fig. 3B and F, and Fig. 4B and F) and Hoechst 33258 (Fig. 3C and G and Fig. 4C and G) triple labelling. Quantification of the number of S-100-positive cells as a percentage of the number of Hoechst-labeled nuclei on the 3rd day of co-culture showed that 76.3 $\pm 5.47 \%$ of BMSCs in SC co-cultures were S-100-positive (Fig. 3D), which was significantly higher than the $3.51 \pm 2.44 \%$ of BMSCs in the acellular control cultures $(\mathrm{P}<0.05)$ (Fig. $3 \mathrm{H}$ and $\mathrm{I})$. Quantification of GFAP-positive cells as a percentage of the number of Hoechst-labeled nuclei showed that $88.7 \pm 3.35 \%$ of BMSCs in SC co-cultures were GFAP-positive, which was significantly higher than the $4.43 \pm 0.99 \%$ of BMSCs in the acellular control cultures $(\mathrm{P}<0.05)$ (Fig. 3J). Quantitative analysis of the number of S-100-positive cells as a percentage of the number of Hoechst-labeled nuclei on the 5th day after co-culture (Fig. 4) showed that $85.6 \pm 1.69 \%$ of BMSCs in SC co-cultures were S-100-positive (Fig. 4D), which was significantly higher than the $3.01 \pm 4.87 \%$ of BMSCs in the acellular control cultures (Fig. 4H). The difference in the percentage of S-100-positive cells was statistically significant at the $\mathrm{P}<0.05$ level (Fig. 4I). Quantification of GFAP-positive cells as a percentage of the number of Hoechst-labeled nuclei showed that 93.1 $\pm 2.27 \%$ of BMSCs in SC co-cultures were GFAP-positive, which was higher than the $4.12 \pm 0.23 \%$ of BMSCs in the acellular control cultures. This difference in GFAP frequency was statistically significant at the $\mathrm{P}<0.05$ level (Fig. 4J).

SC or BMSC growth on the Millicell-HA membrane. The well-grown BMSCs in the upper HA membranes formed an adherent monolayer with a mesenchymal morphology on the 2nd day of co-culture, as shown by hematoxylin and eosin (HE) staining (Fig. 5A), while the BMSCs in the upper HA membranes 

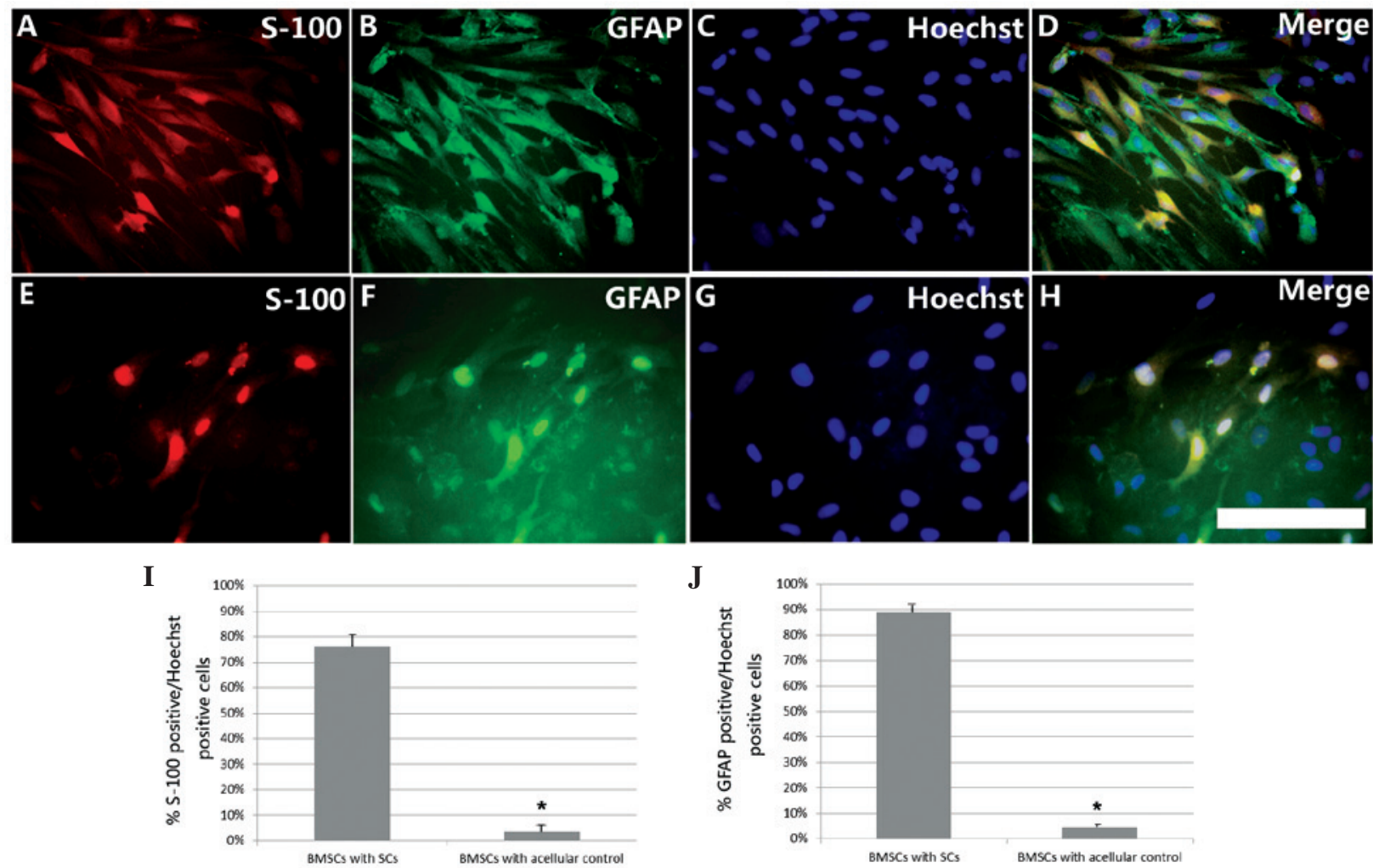

Figure 3. Induction of BMSC differentiation by SC diffusible factors on the 3rd day of co-culture. The glial differentiation of BMSCs was assessed by (A and E) S-100 (red) and (B and F) GFAP (green) immunofluorescence and (C and G) Hoechst nuclear staining (blue). (A-D) Glial differentiation was more evident for BMSCs co-cultured with SCs than for (E-H) BMSCs co-cultured without SCs (scale bar, $50 \mu \mathrm{m}$ ). Quantification of the images provided the percentages of (I) S-100-positive or (J) GFAP-positive cells relative to the number of Hoechst-positive nuclei. Results were averaged for three wells and repeated four times. Each of the four repeats was from a different culture, with each culture in turn being derived from a different animal. Quantitative study showed that the percentage of S-100-positive $(76.3 \pm 5.47 \%, \mathrm{n}=6)$ as well as GFAP-positive $(88.7 \pm 3.35 \%, \mathrm{n}=6)$ cells in BMSCs co-cultured with SCs was significantly higher than that in BMSCs cultured without SCs (S100, 3.51 $\pm 2.44 \%, \mathrm{n}=6$; GFAP, 4.43 $\pm 0.99 \%, \mathrm{n}=6$ ) ( $\mathrm{*}<<0.05)$. BMSC, bone marrow stromal cell; SC, Schwann cell; GFAP, glial fibrillary acidic protein.

exhibited a typical SC-like morphology with spindle-shaped or triangular soma and two or three slender processes on the 5th day of co-culture (Fig. 5B). Similarly, the primary SCs grown in the upper HA membranes developed the typical spindle-shaped soma with two or three slender processes. On the 5th day of co-culture, the SCs grown in the upper HA membranes grew in clusters and their densities were markedly higher than those of the fibroblasts, as shown by HE staining (Fig. 5C).

\section{Discussion}

SC transplantation is a promising therapy for peripheral nerve regeneration; however, the clinical application of SCs is limited due to their low availability (7). Introduction of BMSCs into nerve graft scaffolds represents a novel approach of constructing tissue-engineered nerve grafts owing to their potential advantages in cell-based therapies. However, to date, despite numerous explanations provided by previous studies, experimental evidence is still lacking to support a convincing conclusion regarding the underlying mechanisms of the efficiency of BMSC-based therapy (20). To determine how the implanted BMSCs stimulate peripheral nerve regeneration, the present study established an SC-BMSC co-culturing in vitro model to observe the interaction of BMSCs and SCs using the Millcell-HA system, which consisted of a lower six-well plate and an upper inserted culture plate with an
HA membrane. BMSCs and SCs were seeded into the lower six-well plate or the upper HA membrane of the culture plates. The HA membrane prevented intercellular contact between the SCs and BMSCs (25), allowing the upper and the lower cells to exist in a dynamically synchronous environment, as the pore size $(0.45 \mathrm{~mm})$ of the membrane was small relative to the cell body size of either BMSCs or SCs. The results showed that on the 3rd day of co-culture, the BMSCs co-cultured with SCs differentiated towards glial and SC lineages, and $75 \%$ of BMSCs in the co-culture model were GFAP- and S-100-positive, while the BMSCs in the control culture model hardly showed any GFAP- and S-100-positive populations. However, most of the S-100-and GFAP-positive BMSCs did not acquire the morphology of the adult SCs, which became dense and grew in a longitudinal, parallel fashion. Only a few co-cultured BMSCs showed a typical SC-like morphology, and the majority of them retained their native appearance. In other words, on the 3rd day of co-culture, the BMSCs merely attained the phenotype of SC and glial cells, but not their morphology. In order to validate the glial differentiation of BMSCs in the co-culture system, cells were examined following 5 days of co-culture. Most of the BMSCs co-cultured with SCs not only differentiated towards glial and SC lineages, but also displayed typical SCs-like morphology on the 5th day of co-culture. Therefore, BMSCs not only attained the phenotype of SC and glial 

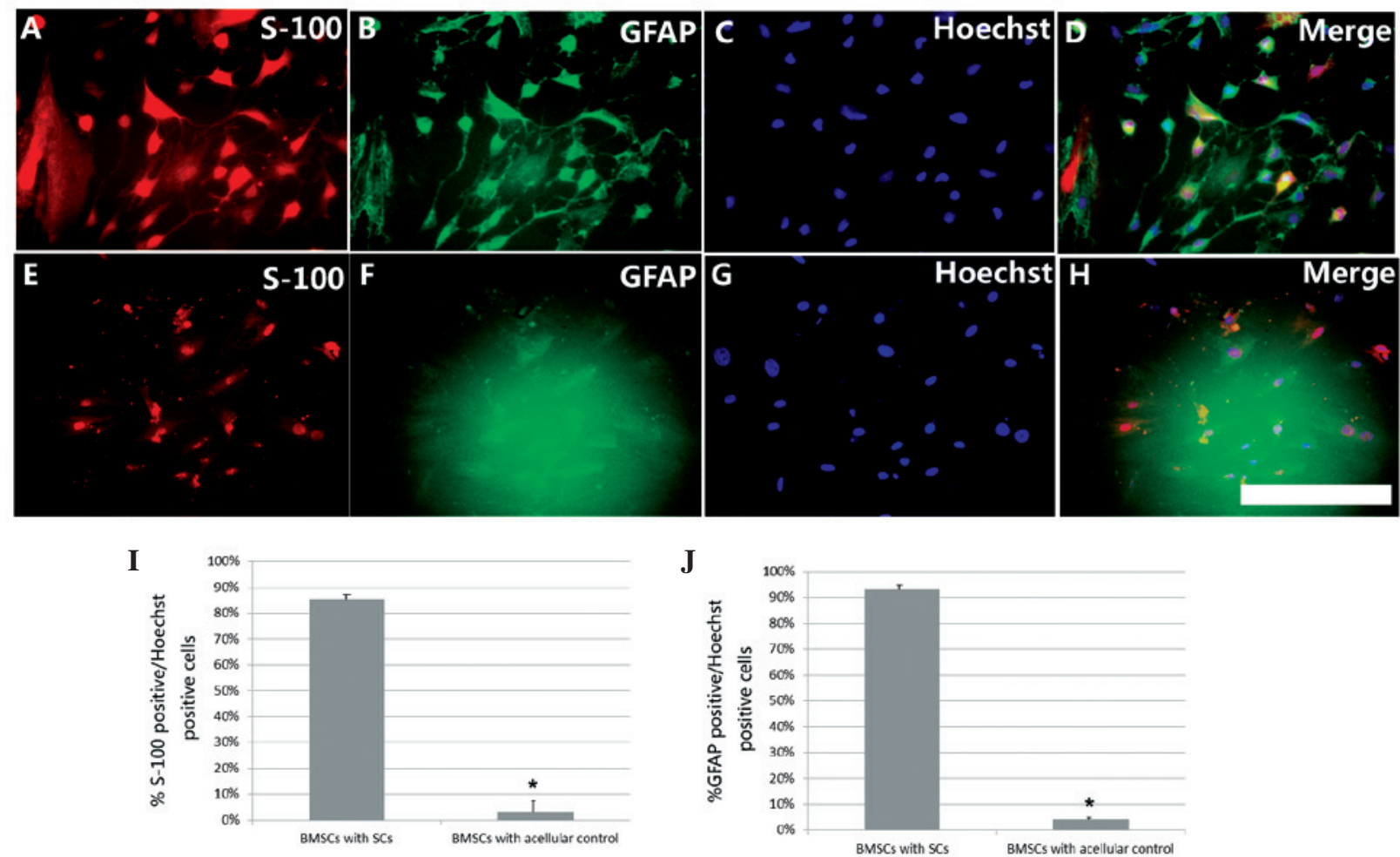

Figure 4. Induction of BMSC differentiation by SC diffusible factors on the 5th day after co-culture. The glial differentiation of BMSCs was assessed by (A and E) S-100 (red) and (B and F) GFAP (green) immunofluorescence and (C and G) Hoechst nuclear staining (blue). (A-D) Glial differentiation was more evident for BMSCs co-cultured with SCs than for (E-H) BMSCs co-cultured with control (scale bar, $50 \mu \mathrm{m}$ ). Quantification of the images provided the percentages of (I) S-100-positive or (J) GFAP-positive cells relative to the number of Hoechst-positive nuclei. Results were averaged for three wells and repeated four times. Each of the four repeats was from a different culture, with each culture in turn being derived from a different animal. Quantitative study showed that the percentage of S-100-positive ( $85.6 \pm 1.69 \%, \mathrm{n}=6)$, as well as GFAP-positive cells $(93.1 \pm 2.27 \%, \mathrm{n}=6)$ in BMSCs co-cultured with SCs was significantly higher than that in BMSCs cultured without SCs ( $\mathrm{S} 100,3.01 \pm 4.87 \%, \mathrm{n}=6$; GFAP, $4.12 \pm 0.23 \%, \mathrm{n}=6)$ ( $\mathrm{P}<0.05)$. BMSC, bone marrow stromal cell; SC, Schwann cell; GFAP, glial fibrillary acidic protein.
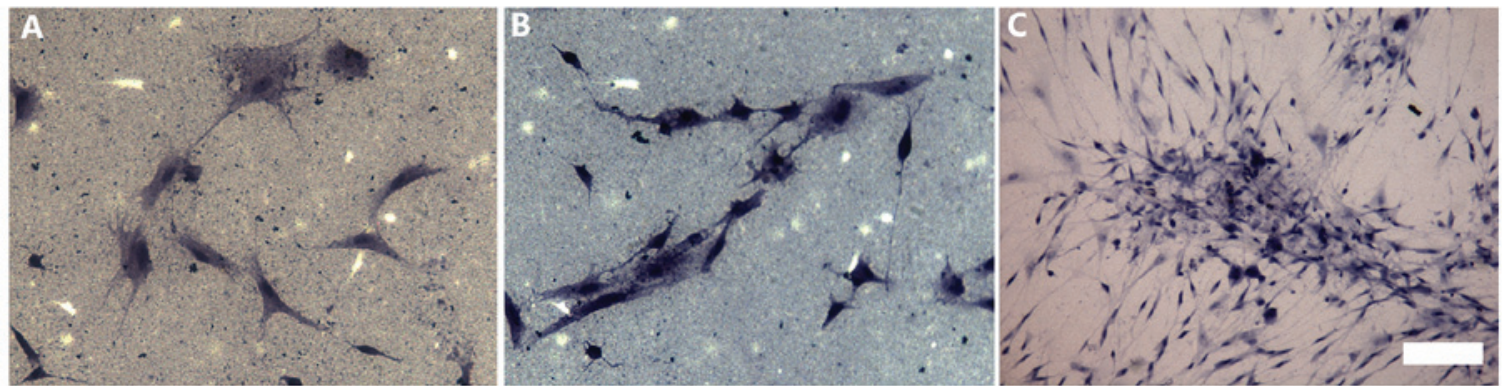

Figure 5. BMSCs as well as SCs grown in the upper HA membranes developed well at the 2nd and 5th day of co-culture, as shown by hematoxylin and eosin staining (scale bar, $200 \mathrm{~mm}$ ). (A) BMSCs were still present an had formed an adherent monolayer with a mesenchymal morphology on the 2nd day of co-culture. (B) BMSCs gradually appeared with an SC-like morphology on the 5th day of co-culture. (C) SCs developed the typical spindle-like shape with a round nucleus in the centre of the cell body and two or three slender processes, and grew in clusters on the 5th day of co-culture. BMSC, bone marrow stromal cell; SC, Schwann cell.

cells, but also their morphology on the 5th day of co-culture. Quantitative evaluation on the 3rd or 5th day of co-culture showed that the percentage of S-100 and GFAP-positive BMSCs in SC co-culture was significantly higher than that of BMSCs in the acellular control cultures. It is thought that the differentiation of implanted BMSCs into SCs is the most important event in the beneficial effect of BMSCs since peripheral nerve system regeneration occurs mainly through a series of events produced by activated SCs (24). Previous studies by our group also proved that peripheral nerve defects in the rhesus monkeys could be repaired with chemically extracted allogenic nerves implanted with cultured autologous undifferentiated BMSCs $(24,26,27)$. Numerous studies have also shown that differentiated and undifferentiated adult BMSCs exert a beneficial effect on injured peripheral nerves $(19,21-24,28,29)$. It is possible that with time, they attained either an SC-like functional or morphological phenotype after transplantation. Previous studies have demonstrated that adult SCs can release neurotrophic factors, extracellular matrix and cell adhesion 
molecules $(24,30)$, which have been shown to induce neural or SC-like differentiation of BMSCs $(19,21,23,24,26-28,31)$. Thus, it is possible that the differentiation of BMSCs in the present study may have been the effect of one or more of the soluble factors released by the co-cultured SCs. An alternative explanation for the differentiation of BMSCs is that it could not be completely excluded that the favorable cellular processes may have been able to penetrate the HA membrane to physically interact with BMSCs in the six-well plate (24). The present study also found that the densities of BMSCs were significantly higher in SC co-cultures than those in the acellular control cultures on the 3rd as well on the 5th day of co-culture. The above results illustrated that SCs had beneficial effects on BMSCs in a dynamic and contact-free co-culture model. The transdifferentiation mechanism in BMSCs-based therapy is thought to be due to real transdifferentiation rather than spontaneous fusion of BMSCs with host cells $(32,33)$. In addition, the present study investigated in vitro effects of adult rat BMSCs on adult rat SC proliferation, which has not been reported in any previous studies, to the best of our knowledge. As demonstrated by labeling with S-100 antibodies and Hoechst 33342, SCs co-cultured with BMSCs exhibited an increase in their proliferation as compared to that of SCs cultured alone. An explanation of this outcome is that BMSCs were able to produce neurotrophic factors, cytokines, extracellular matrix components and supporting substances, which may have activated host SCs (24,31,34-37). This may explain why the BMSCs promoted the proliferation of adult SCs in vitro without intercellular contact in the present study. Another interesting finding was that the proportion of S-100-positive cells in primary cultures of SCs was $\sim 70 \%$, while it was only $30 \%$ in the acellular control group in the co-culture model. It is possible that this discrepancy in results (70 vs. 30\%) is due to differential time-points of measurement, as the primary culture group was assessed on day 5 and the acellular control group in the co-culture model was assessed on day 10 of culture. Previous studies have shown that fibroblast populations dominate SC culture with the elongation of culture time $(24,37)$. While the proportion of S-100-positive SCs in the BMSCs co-culture model is $70 \%$ at 10 days of culture, which equal to the proportion of S-100-positive cells in primary cultures of SCs at 5 days of culture. Collectively, the results of the present study suggested the beneficial effects of BMSCs on the survival and proliferation of SCs in vitro. To verify whether an interaction between BMSCs and SCs is present, the growth of BMSCs or SCs on the HA membrane was investigated. BMSCs on the upper HA membrane grew well and formed an adherent monolayer with a mesenchymal morphology on the 2nd day of co-culture, while on the 5th day of co-culture, the BMSCs on the upper HA membrane exhibited a typical SC-like morphology, which was consistent with the change of BMSCs in the lower six-well plate in the co-culture model on the 5th day of co-culture. The primary SCs grown on the upper HA membrane displayed the typical spindle-shaped soma with two or three slender processes. On the 5th day of co-culture, the SCs grown on the upper HA membrane grew in clusters and their density was markedly higher than that of the fibroblasts, which was consistent with the observations on the SCs in the lower six-well plate in the co-culture system on the 5th day of co-culture.
In conclusion, the present in vitro study using co-culture methods demonstrated that adult BMSCs enhanced the proliferation of adult SCs, and that adult SCs promoted the Schwann-like cell differentiation of adult BMSCs. These results demonstrated the beneficial reciprocal effects of adult BMSCs and adult SCs in a dynamic and cell-cell contact-free co-culture system.

\section{Acknowledgements}

This work was supported by the National Natural Science Foundation of China (grant no. 81401022) and Guangdong Provinical Science and Technology Projects (grant no. 2014A020211026).

\section{References}

1. Bunge RP: The role of the Schwann cell in trophic support and regeneration. J Neurol 242 (Suppl 1): S19-S21, 1994.

2. Jessen KR and Mirsky R: Developmental regulation in the Schwann cell lineage. Adv Exp Med Biol 468: 3-12, 1999.

3. Schlosshauer B, Müller E, Schröder B, Planck H and Müller HW: Rat Schwann cells in bioresorbable nerve guides to promote and accelerate axonal regeneration. Brain Res 963: 321-326, 2003.

4. Brenner MJ, Lowe JB III, Fox IK, Mackinnon SE, Hunter DA, Darcy MD, Duncan JR, Wood P and Mohanakumar T: Effects of Schwann cells and donor antigen on long-nerve allograft regeneration. Microsurgery 25: 61-70, 2005.

5. Keilhoff G, Goihl A, Langnäse K, Fansa H and Wolf G: Transdifferentiation of mesenchymal stem cells into Schwann cell-like myelinating cells. Eur J Cell Biol 85: 11-24, 2006.

6. Baksh D, Song L and Tuan RS: Adult mesenchymal stem cells: Characterization, differentiation and application in cell and gene therapy. J Cell Mol Med 8: 301-316, 2004.

7. Wei Y, Gong K, Zheng Z, Liu L, Wang A, Zhang L, Ao Q, Gong Y and Zhang X: Schwann-like cell differentiation of rat adipose-derived stem cells by indirect co-culture with Schwann cells in vitro. Cell Prolif 43:606-616, 2010.

8. Pittenger MF, Mackay AM, Beck SC, Jaiswal RK, Douglas R, Mosca JD, Moorman MA, Simonetti DW, Craig S, Marshak DR: Multilineage potential of adult human mesenchymal stem cells. Science 284: 143-147, 1999.

9. Ladak A, Olson J, Tredget EE and Gordon T: Differentiation of mesenchymal stem cells to support peripheral nerve regeneration in a rat model. Exp Neurol 228:242-252, 2011.

10. Phinney DG, Kopen G, Isaacson RL and Prockop DJ: Plastic adherent stromal cells from the bone marrow of commonly used strains of inbred mice: Variations in yield, growth, and differentiation. J Cell Biochem 72: 570-585, 1999.

11. Azizi SA, Stokes D, Augelli BJ, DiGirolamo C and Prockop DJ: Engraftment and migration of human bone marrow stromal cells implanted in the brains of albino rats-similarities to astrocyte grafts. Proc Natl Acad Sci USA. 95: 3908-3913, 1998.

12. Deng W, Obrocka M, Fischer I and Prockop DJ: In vitro differentiation of human marrow stromal cells into early progenitors of neural cells by conditions that increase intracellular cyclic AMP. Biochem Biophys Res Commun 282: 148-152, 2001.

13. Sanchez-Ramos J, Song S, Cardozo-Pelaez F, Hazzi C, Stedeford T, Willing A, Freeman TB, Saporta S, Janssen W, Patel N, et al: Adult bone marrow stromal cells differentiate into neural cells in vitro. Exp Neurol 164: 247-256, 2000.

14. Woodbury D, Schwarz EJ, Prockop DJ and Black IB: Adult rat and human bone marrow stromal cells differentiate into neurons. J Neurosci Res 61: 364-370, 2000.

15. Lu L, Chen X, Zhang CW, Yang WL, Wu YJ, Sun L, Bai LM, Gu XS, Ahmed S, Dawe GS, et al: Morphological and functional characterization of predifferentiation of myelinating glia-like cells from human bone marrow stromal cells through activation of F3/Notch signaling in mouse retina. Stem Cells 26: 580-590, 2008.

16. Mahay D, Terenghi G and Shawcross SG: Schwann cell mediated trophic effects by differentiated mesenchymal stem cells. Exp Cell Res 314: 2692-2701, 2008a. 
17. Mahay D, Terenghi G and Shawcross SG: Growth factors in mesenchymal stem cells following glial-cell differentiation. Biotechnol Appl Biochem 51:167-176, 2008b.

18. Caddick J, Kingham PJ, Gardiner NJ, Wiberg M and Terenghi G: Phenotypic and functional characteristics of mesenchymal stem cells differentiated along a Schwann cell lineage. Glia 54: 840-849, 2006.

19. Dezawa M, Takahashi I, Esaki M, Takano M and Sawada H: Sciatic nerve regeneration in rats induced by transplantation of in vitro differentiated bone-marrow stromal cells Eur J Neurosci 14: 1771-1776, 2001.

20. Wang J, Ding F, Gu Y, Liu J and Gu X: Bone marrow mesenchymal stem cells promote cell proliferation and neurotrophic function of Schwann cells in vitro and in vivo. Brain Res 1262: 7-15, 2009.

21. Hou SY, Zhang HY, Quan DP, Liu XL and Zhu JK: Tissue-engineered peripheral nerve grafting by differentiated bone marrow stromal cells. Neuroscience 140: 101-110, 2006.

22. Mimura T, Dezawa M, Kanno H, Sawada H and Yamamoto I: Peripheral nerve regeneration by transplantation of bone marrow stromal cell-derived Schwann cells in adult rats. J Neurosurg 101: 806-812, 2004.

23. Cuevas P, Carceller F, Dujovny M, Garcia-Gómez I, Cuevas B, González-Corrochano R, Diaz-González D and Reimers D: Peripheral nerve regeneration by bone marrow stromal cells. Neurol Res 24: 634-638, 2002.

24. Zhou LN, Zhang JW, Wang JC, Lei WL, Liu XL and Zhou LH: Bone marrow stromal and Schwann cells from adult rats can interact synergistically to aid in peripheral nerve repair even without intercellular contact in vitro. J Tissue Eng Regen Med 6 : 579-588, 2012.

25. Zurita M, Vaquero J, Oya S, Bonilla C and Aguayo C: Neurotrophic Schwann-cell factors induce neural differentiation of bone marrow stromal cells. Neuroreport 18 : 1713-1717, 2007

26. Hu J, Zhu QT, Liu XL, Xu YB and Zhu JK: Repair of extended peripheral nerve lesions in rhesus monkeys using acellular allogenic nerve grafts implanted with autologous mesenchymal stem cells. Exp Neurol 204: 658-666, 2007.
27. Wang D, Liu XL, Zhu JK, Jiang L, Hu J, Zhang Y, Yang LM, Wang HG and Yi JH: Bridging small-gap peripheral nerve defects using acellular nerve allograft implanted with autologous bone marrow stromal cells in primates. Brain Res 1188: 44-53, 2008.

28. Akiyama Y, Radtke C, Honmou O and Kocsis JD: Remyelination of the spinal cord following intravenous delivery of bone marrow cells. Glia 39: 229-236, 2002

29. Dezawa M, Kanno H, Hoshino M et al: Specific induction of neuronal cells from bone marrow stromal cells and application for autologous transplantation. J Clin Invest 113: 1701-1710, 2004.

30. Lin $\mathrm{CH}$, Cheng FC, Lu YZ, Chu LF, Wang CH, Hsueh CM: Protection of ischemic brain cells is dependent on astrocyte-derived growth factors and their receptors. Exp Neurol 201: 225-233, 2006.

31. Chen CJ, Ou YC, Liao SL, Chen WY, Chen SY, Wu CW, Wang CC, Wang WY, Huang YS and Hsu SH: Transplantation of bone marrow stromal cells for peripheral nerve repair. Exp Neurol 204: 443-453, 2007.

32. Weimann JM, Charlton CA, Brazelton TR, Hackman RC and Blau HM: Contribution of transplanted bone marrow cells to Purkinje neurons in human adult brains. Proc Natl Acad Sci USA 100: 2088-2093, 2003a.

33. Weimann JM, Johansson CB, Trejo A and Blau HM: Stable reprogrammed heterokaryons form spontaneously in Purkinje neurons after bone marrow transplant. Nat Cell Biol 5: 959-966, 2003b.

34. Chopp M and Li Y: Treatment of neural injury with marrow stromal cells. Lancet Neurol 1: 92-100, 2002.

35. Lee AC, Yu VM, Lowe JB III, Brenner MJ, Hunter DA, Mackinnon SE and Sakiyama-Elbert SE: Controlled release of nerve growth factor enhances sciatic nerve regeneration. Exp Neurol 184: 295-303, 2003.

36. Marcol W, Kotulska K, Swiech-Sabuda E, Larysz-Brysz M, Gołka B, Górka D and Lewin-Kowalik J: Regeneration of sciatic nerves of adult rats induced by extracts from distal stumps of pre-degenerated peripheral nerves. J Neurosci Res 72: 417-424, 2003.

37. Neuhuber B, Timothy Himes B, Shumsky JS, Gallo G and Fischer I: Axon growth and recovery of function supported by human bone marrow stromal cells in the injured spinal cord exhibit donor variations. Brain Res 1035: 73-85, 2005. 